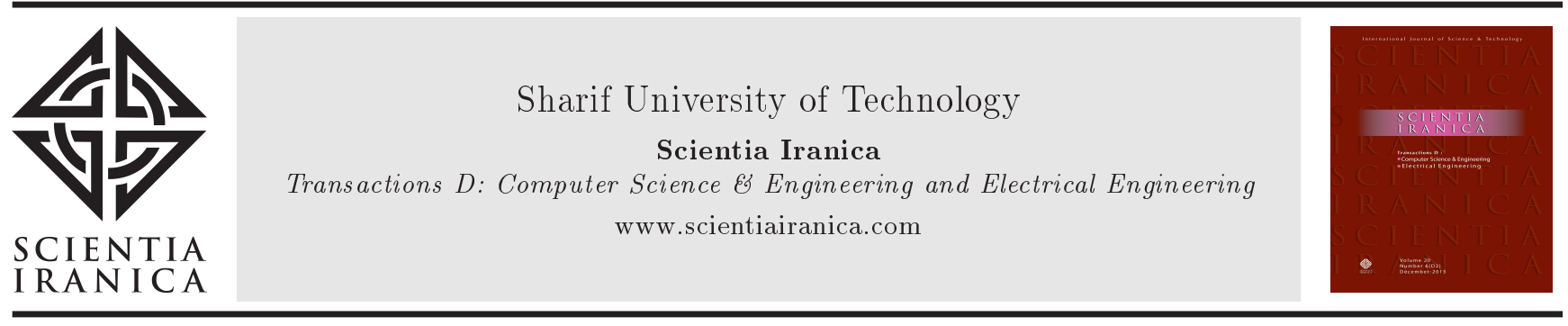

\title{
A proposed maximum power point tracking by using adaptive fuzzy logic controller for photovoltaic systems
}

\author{
A. Gheibi*, S.M.A. Mohammadi and M. Maghfoori Farsangi \\ Department of Electrical Engineering, Shahid Bahonar University of Kerman, Kerman, Iran.
}

Received 22 September 2014; received in revised form 26 June 2015; accepted 5 October 2015

\section{KEYWORDS}

Adaptive fuzzy logic control;

Boost converter;

Conventional fuzzy

controller;

Decision-making;

Real-time regulation;

Solar cell.

\begin{abstract}
This paper presents a proposed method to search Maximum Power Point (MPP) based on the Adaptive Fuzzy Logic Control (AFLC), which is applied to photovoltaic $(\mathrm{PV})$ systems under varying temperatures and radiations. The proposed system is composed of boost converter, two fuzzy controllers and load. Whenever environmental conditions change in wide range, using only Conventional Fuzzy Logic Controller (CFLC) is not adequate and causes more errors in tracking. The proposed AFLC comprises two stages: Online and Offline tuning. The offline method, by accurately setting CFLC controller parameters, is applied for relatively stable atmospheric conditions. Meanwhile, the online method is considered for unstable atmospheric conditions and contains two fuzzy controllers - one primary, one secondary. The primary fuzzy controller is the CFLC, and the secondary controller is the decision-making, which due to atmospheric conditions, alters the primary fuzzy controller parameters in order to achieve a better answer compared to utilizing CFLC. Decision-making controller with changing in irradiation and temperature changes gain of inputs of CFLC, simultaneously, that it increases rate and accuracy of tracking in comparison with using only fuzzy controller. By simulating results using CFLC and AFLC controllers, the proposed method is able to improve performance indicators with respect to CFLC.
\end{abstract}

(C) 2016 Sharif University of Technology. All rights reserved.

\section{Introduction}

Utilizing solar cells provides many advantages including no fuel cost, no pollution, and no need for maintenance equipment. Although having initial high costs as compared to other energy generating equipment and, therefore, not so good public prosperity wording them, however, with the development of solar cell technology, their prices have dropped significantly [1]. Solar cells constitute PN junctions, which are composed of electrons and holes. Movement of these electrons and

* Corresponding author. Tel./Fax: 066-33228124 E-mail addresses: a.gheibi907@gmail.com (A. Gheibi); s_m_ali_mohammadi@yahoo.com (S.M.A. Mohammadi); mmaghfoori@mail.uk.ac.ir (M. Maghfoori Farsangi) holes inside the load connected to the cell creates an electric current and, finally, an electric power on the photovoltaic (PV) output terminals. A photovoltaic array is formed by the series and parallel connections of solar cells; in these states, higher electrical power can be obtained. The electrical power obtained from the photovoltaic array is not constant and changes due to temperature change, radiation, and changes in the amount of load. Hence, considering changes in the effective parameters of the obtained power from the photovoltaic generator, any change in the previously mentioned factors exhibits a new maximum point on the power-voltage curve. In order to obtain this maximum point, many innovative methods have been introduced. All these methods take advantage of the fact that the power-voltage curve slope has a 
value of zero in the maximum point. In a generalized classification, these methods can be divided into five main categories:

A. Control algorithm: In this method, the point of maximum power is obtained by measuring voltage and photovoltaic power using a simple algorithm. The P\&O and IC methods are two of the most popular and recognized methods in this regard [2,3];

B. Control variable: Tracking is carried out by measuring the point of maximum power inside each control loop. In this method, voltage (current or power) is considered to be control variable. By setting and adapting voltage with one desirable voltage (current or power), by which maximum power has occurred, the working point of the array is maintained near MPP [4-7];

C. Math-based methods: Utilizing photovoltaic current and voltage relations, and keeping in mind that derivative of power against voltage (current) must equal zero, a non-linear equation is obtained, by which results can be obtained using numerical methods. The result of this equation gives the voltage (current) at the point of maximum power [8,9];

D. Intelligent control: In this method, decisions are made by the controller based on zero-error (derivative of power relative to voltage (current)). Among cases carried out using this method are fuzzy [1012], adaptive fuzzy [13], neural networks [14];

E. Hybrid method: By combining one or more of the methods listed above, the performance of the controller can improve [15].

In the future, for decreasing complexity of the system, the methods can be used as they lead to less complexity in system, i.e. TSK fuzzy [16]. Also, the system can be applied with fuzzy logic based on classical methods in order to ensure stability of the system [17].

Fuzzy method is one of the best methods because of its suitable answer to the uncertainty present in the system, and its desirable speed and precision. This method gives suitable and desirable answers even with limited changes in system parameters. However, if the changes in system parameters are extended (e.g., extensive changes in radiation or ambient temperature or load in a short period), tracking the point of maximum power will experience severe errors. DC or AC loads are considered constant in stand-alone photovoltaic systems. Meanwhile, if the load constantly changes in time (like temperature and radiation), we will practically have three time-varying parameters, which make designing the controller even more difficult. Therefore, in this article in order to prevent further intricacy in the system, we assume that the load connected to the DC system is constant. In adaptive fuzzy methods, fuzzy controller parameters are set in offline mode in order to adapt with these conditions [13]. Whereas if radiation and temperature are extended in a non-predictive manner (unstable atmospheric conditions), we will require resetting the fuzzy controller parameter, which in some cases is impossible. The proposed method includes two stages: Offline and Online. In the offline method, if changes are limited and predictive, precise setting of fuzzy controller parameters is possible. However, in unpredicted cases with varied ranges of temperature and radiation, fuzzy controller parameters must be set using the online mode.

Online setting of fuzzy controller parameters is a timely and complex process. In this state, the time assigned for setting controller parameters must be less than the time of temperature and radiation intensity change. Otherwise, tracking will not be proper and the response obtained in this state may even be worse than the fuzzy method. In order to prevent this situation, we have conducted the CFLC parameter setting in a different manner. In this state, we have added a second fuzzy controller, namely "decision-making", to the system. The decision-making controller is a fuzzy controller, which constitutes temperature and radiation intensity as inputs. The output of this controller changes the primary fuzzy controller parameters proportional with these instantaneous changes in order to obtain better responses compared to the fuzzy method. Simulations have been carried out using MATLAB/Simulink software. By comparing the proposed method with the fuzzy method, in simultaneous temperature and radiation change conditions, simulation results show that the proposed method follows the maximum point of power with better speed and precision.

\section{PV generator and boost converter}

As mentioned earlier, the relationship between output voltage and power of the PV at varying light intensities and temperatures is non-linear as depicted in Figure 1.

At the same irradiation and temperature, there is a unique point located at the knee of the power $(P)$-voltage $(V)$ curve that is called Maximum Power Point (MPP). The design of a solar energy system is generally concerned with obtaining maximum efficiency at minimum cost. In a solar cell operating under the normal conditions, even a small derivation of $1 \%$ from the optimum power transfer condition can cause a loss of output power by nearly $10 \%$ [18]. For increasing efficiency of $\mathrm{PV}$, we need to track MPP.

A proposed Maximum Power Point Tracking (MPPT) scheme obtained by varying the duty ratio for DC/DC boost converter has been successful $[19,20]$. The Boost converters are extremely used in Photovoltaics in order to keep voltage at a value that has maximum power [21]. 


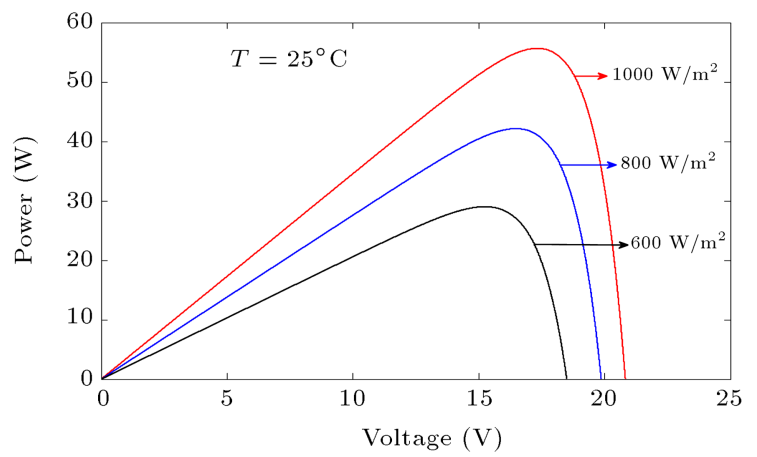

(a)

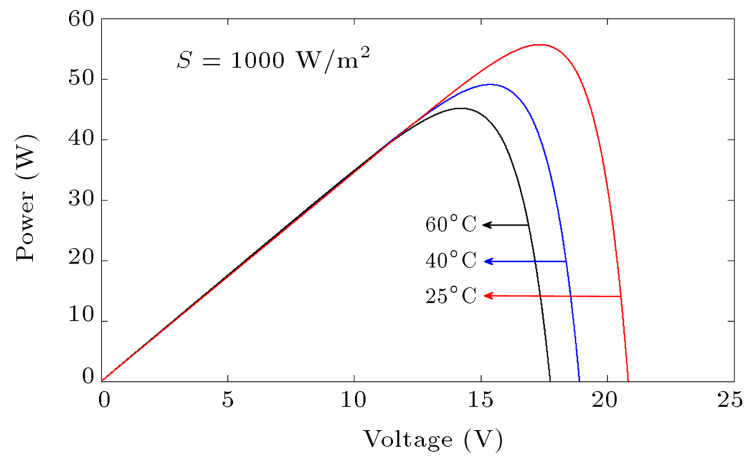

(b)

Figure 1. Changes of Maximum Power Point (MPP): (a) Irradiation; and (b) temperature. $T$ is temperature and $S$ is irradiation.

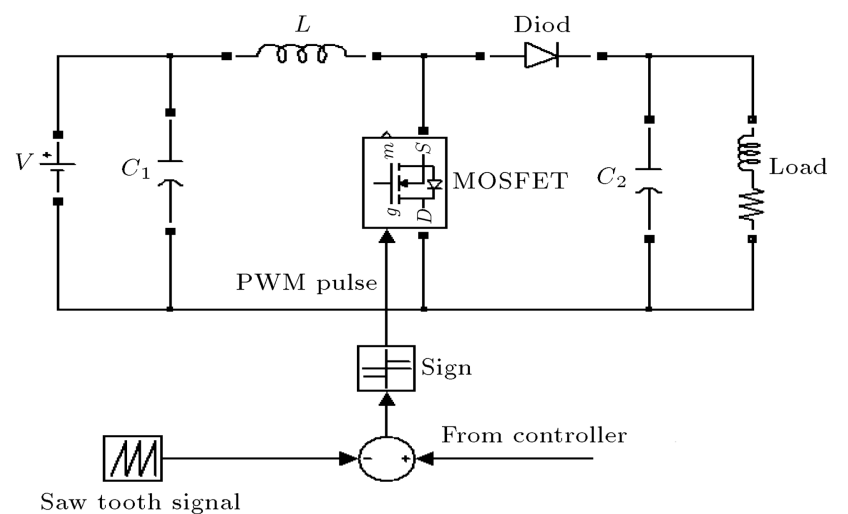

Figure 2. Boost converter with PWM (Pulse Wide Modulation) comparator.

Figure 2 shows boost converter and PWM comparator. PWM comparator is applied to compare the command signal from controller with a saw tooth signal for making PWM control signal at the gate of MOSFET. In the MPP point, the output photovoltaic resistance is $R_{\mathrm{MPP}}$, which has a small value, and a boost converter is used in order to reach maximum system performance. The boost converter can change input resistances larger than to equal to $R_{\mathrm{MPP}}$ and considering the fact that photovoltaic resistance is stronger than $R_{\mathrm{MPP}}$ resistance in high radiations (near $1000 \mathrm{~W} / \mathrm{m}^{2}$ ), and noticing that during daytime- tracking, photovoltaic resistance is usually higher than $R_{\mathrm{MPP}}$ resistance, the boost converter is employed more often for tracking purposes in photovoltaics [22].

\section{Conventional fuzzy logic controller}

Conventional Fuzzy Logic Controller (CFLC) is made of three units: fuzzification, knowledge and inference unit, and defuzzification as shown in Figure 3 [23,24].

In the knowledge and inference unit there are several rules that connect the fuzzy output to the fuzzy inputs by understanding the system behavior. The process of defuzzification is the last step for designing the fuzzy control algorithm, which calculates the crisp output of the fuzzy control. Implementation of the type 1 fuzzy controller has two input variables; the error $e(t)$ and the error derivative $d e(t)$, In MPP:

$$
e(t)=\frac{d P(t)}{d V(t)}=\frac{d(V I)}{d V}=I+V\left(\frac{d I}{d V}\right)=0,
$$

then:

$$
\begin{aligned}
& e(t)=\frac{\Delta P(t)}{\Delta V(t)}, \\
& \frac{d e(t)}{d t} .
\end{aligned}
$$

The input $e(t)$ indicates the error, which the load operation point makes at the moment ' $t$ ' that if $e(t)<$ 0 , the operation point is located on the rightand if $e(t)>$ 0 , it is located on the left of the MPP characteristic; furthermore, $d e(t)$ shows the moving direction of this point.

\section{Characteristics of controllers}

To adapt a behavior means to alter it and to reach a new state; thus, an adaptive controller is a controller that its behavior in response to modifying in the dynamics of the process can change [25]. The non-linear nature of solar cell system requires a kind of controller which not only works appropriately at the constant temperature conditions and irradiation, based on which the controller is designed, but also has an acceptable function at the variable temperature conditions, as well as the irradiation close to design conditions. However, if the condition variations are wide, the controller parameters proportionate to such variations should be updated. Adaptive fuzzy logic controller consists of two controllers, CFLC and decision-making. Figure 4 shows the system under control with the proposed AFLC.

\section{1. $C F L C$}

Membership function of inputs and output is shown in Figure 5. CFLC consists of 25 rules which are 


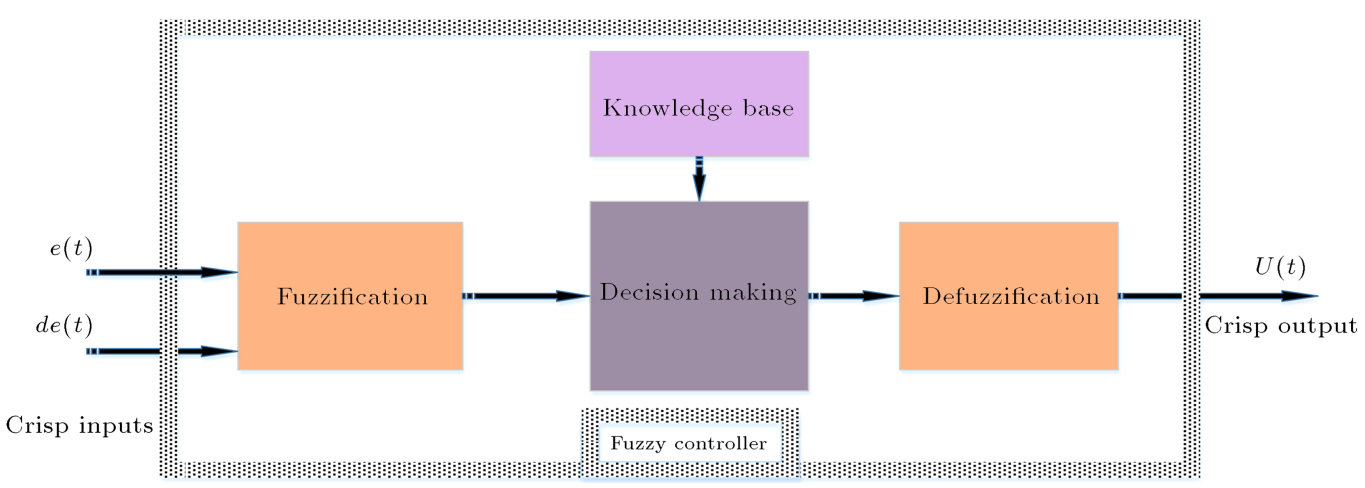

Figure 3. Structure of a type1 fuzzy logic controller.

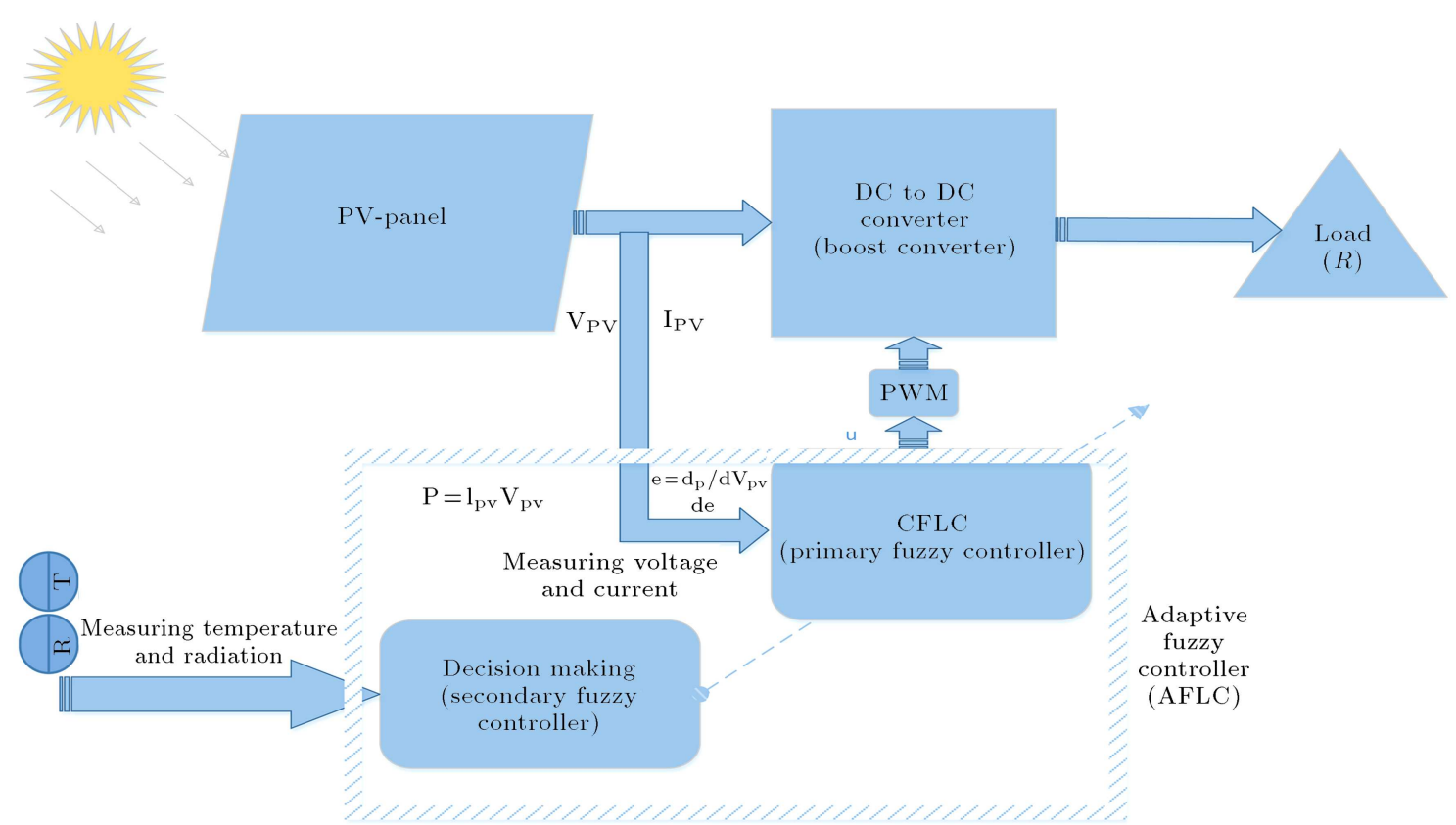

Figure 4. Structure of a photovoltaic system under control.

Table 1. Rule base of Conventional Fuzzy Logic Controller (CFLC).

\begin{tabular}{cccccc}
\hline \multirow{2}{*}{ PB } & \multicolumn{5}{c}{$\boldsymbol{d}(\mathbf{t})$} \\
\cline { 2 - 6 } & $\boldsymbol{e}(\boldsymbol{t})$ & NB & NS & ZE & PS \\
\hline ZE & NB & NS & NS & NB & PS \\
ZE & NS & NS & ZE & NS & ZE \\
PS & ZE & NS & ZE & ZE & ZE \\
PB & PS & ZE & ZE & PS & PS \\
PB & PB & ZE & PS & PB & PS \\
\hline
\end{tabular}

observed in Table 1. CFLC has a good performance in environmentally fixed condition and even somewhat in variable conditions [10]. But, if this change leads to sudden change in output power, then the controller cannot trace MPP in some operation points. For increasing efficiency of MPPT, another fuzzy controller is added to PV system to improve system performance, which is called decision-making.

\subsection{Decision-making}

Decision-making consists of a fuzzy controller with two temperature and irradiation inputs and output gain. The gain changes proportional with the inputs of CFLC, which it will compensate drop of errors of CFLC according to Figure 6. According to the standard conditions in Table 2 and after selection of an appropriate criterion for tracking, to obtain the desired response at any temperature conditions and irradiation, the gain rate of the control signal of fuzzy controller should be regulated.

However, if the criterion function condition is not satisfied, the interval membership functions should be regulated on it. Figure 7 shows the flowchart of the regulation of gain rates and membership functions to regulate the control signal of the controller.

According to the desired response, which is obtained in standard conditions, the appropriate tracking criterion is defined as follows: 
Table 2. The defined standard condition for a photovoltaic (PV) system.

\begin{tabular}{cccccc}
\hline Parameters & Irradiation & Temperature & Maximum power & $\begin{array}{c}\text { Open } \\
\text { circuit } \\
\text { current }\end{array}$ & $\begin{array}{c}\text { Short circuit } \\
\text { voltage }\end{array}$ \\
\hline Standard condition & $1000 \mathrm{~W} / \mathrm{m}^{2}$ & $25^{\circ} \mathrm{C}$ & $55 \mathrm{w}$ & $3.45 \mathrm{~A}$ & $21.2 \mathrm{~V}$ \\
\hline
\end{tabular}

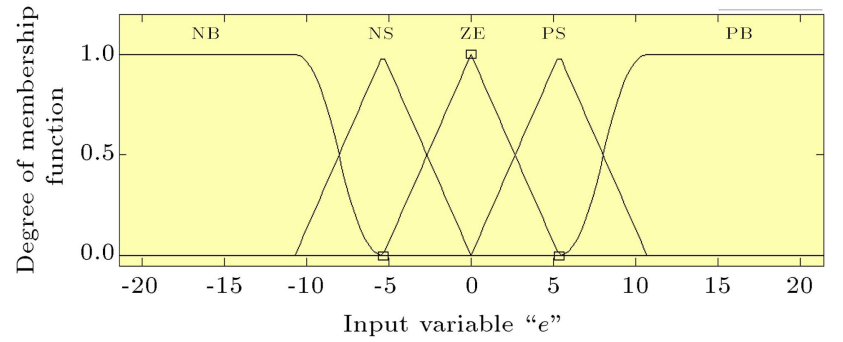

(a)

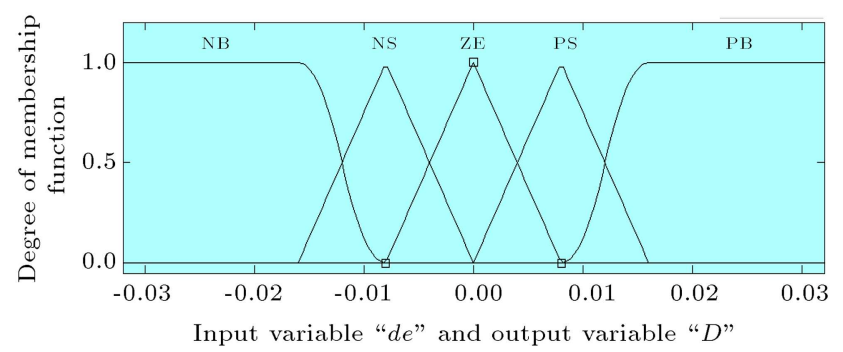

(b)

Figure 5. Membership function: (a) Input of $e(t)$; and (b) input of $d e(t)$ and output of $D(t)$.

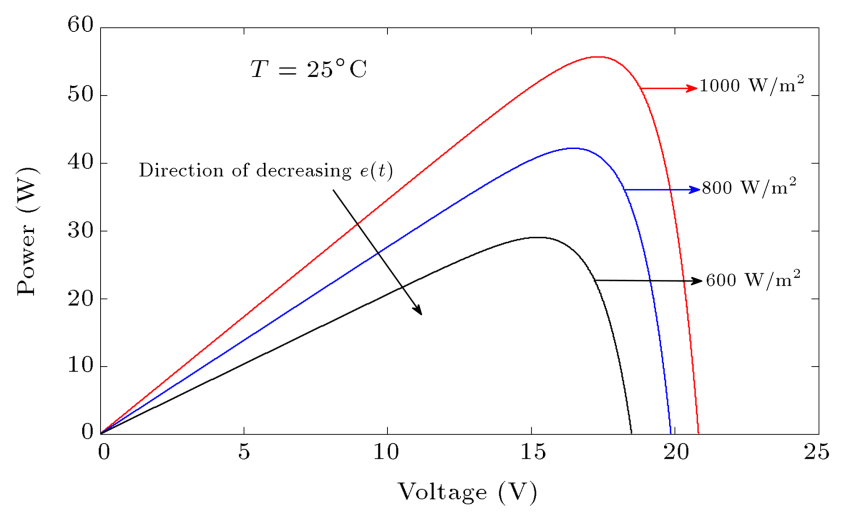

Figure 6. Direction of decreasing $e(t)$ with changing irradiation; $T$ is temperature.

$$
\text { Fit }=\left(M_{m}+4(\mathrm{SE}+V)\right) t_{p} / 2,
$$

where:

$$
M_{m}=\max _{t}(P(t))-\sum_{i=t_{\max }}^{t} P(i) / N
$$

where, $M_{m}$ is the difference between the maximum power and the average values of power and the maximum power to the end of the simulation time with a fixed sampling frequency, $N$ is the number of samplings of maximum power to the end of simulation time, $V$ is

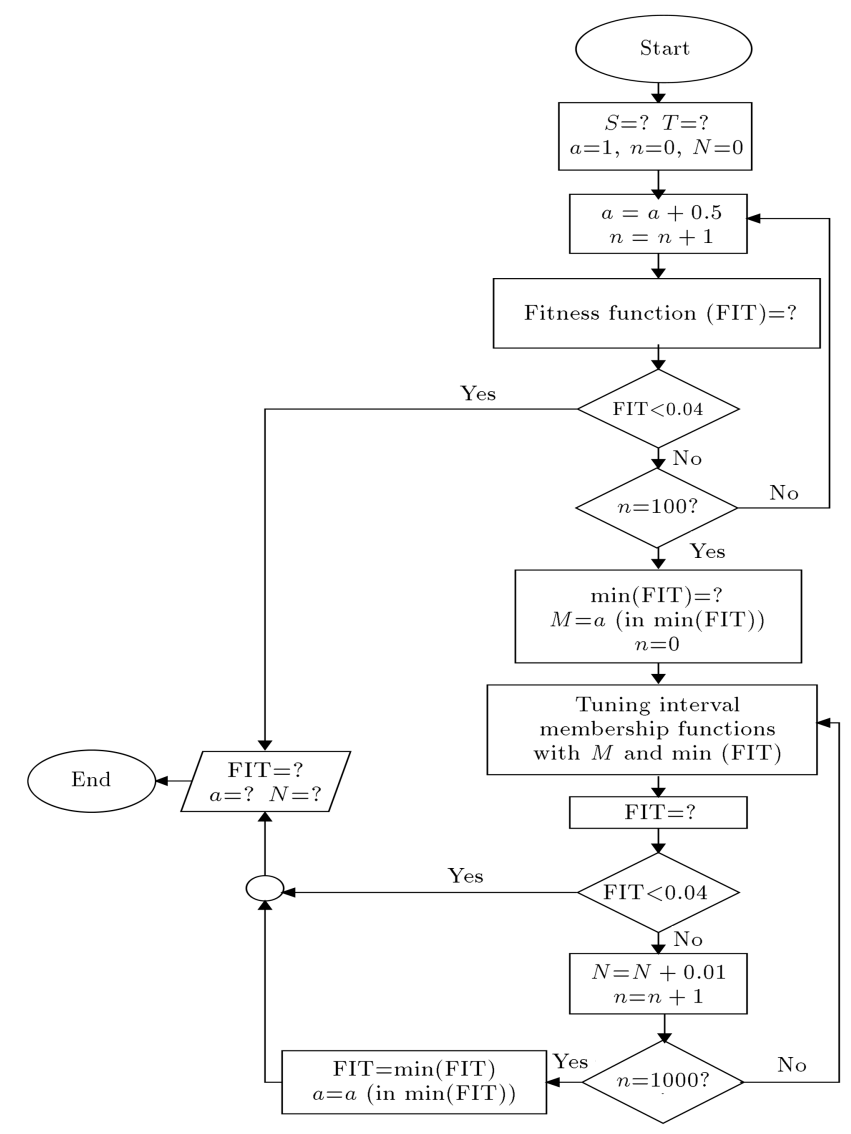

Figure 7. Flowchart of search values of gain and interval movement of membership function for a given irradiation and temperature, where $a$ is gain of controller, $N$ is value of interval movement membership function, $n$ is value iteration loop, $T$ is temperature, and $S$ is irradiation.

the photovoltaic output power variance, $t_{p}$ is the time at the maximum power point, and $\mathrm{SE}$ is defined as follows:

$$
\mathrm{SE}=\int_{0}^{t}\left|e\left(t^{\prime}\right)\right| d t^{\prime} .
$$

$M_{m}$ and $V$ indicate the values of tracking and power variance, which present the fluctuation values around the maximum power, and $t_{p}$ indicates the tracking rate. Criterion function is designed to satisfy the following conditions:

a) Acceptable fluctuation around the maximum point: up to $8 \%$ of the maximum power;

b) Tracking error rate: less than 1 ; 
c) Acceptable response rate: the time at maximum power point $\left(t_{p}\right)$ less than 0.02 second.

Based upon measurements performed on the optimal values for Eq. (3), Fit $<0.04$; thus, in the performed simulations, the algorithm's stop condition for the best answer is considered less than 0.04. In cases where this condition is not met, the best answer is considered to be in a Cycle of Repetition.

\section{Off-line regulation}

The first discussion presented here is about the changes that the power-voltage characteristic curve undergoes due to the changes in the environmental conditions, compared with the standard mode. With regard to Figure 6, which shows the changes in the powervoltage curve based on the irradiation changes, it can be observed that the value of fuzzy controller error input will decrease, compared with the standard conditions $\left(1000 \mathrm{~W} / \mathrm{m}^{2}\right)$, by reducing the amount of irradiation, and this loss can be compensated by considering the appropriate gain for the values of fuzzy controller input errors. Figure 8 represents the changes in the power-voltage characteristic curve according to the temperature changes compared with the standard mode of $25^{\circ} \mathrm{C}$.

It can be observed that at the first area, the voltage increases at MPP by decreasing temperature and decreases at MPP by temperature increase. Moreover, by reducing the irradiation from $100 \mathrm{~W} / \mathrm{m}^{2}$, the temperature will decrease by $3^{\circ} \mathrm{C}$.

\subsection{Regulation of gain}

In this case, according to the flowchart of Figure 7 and noting the required codes, for some irradiation modes and for each mode with a few temperature changes, we calculate the range of gain changes for different irradiations and temperatures.

\subsection{Regulation of membership functions}

If the conditions of Eq. (3) are not met, we regulate the interval membership functions for improving the

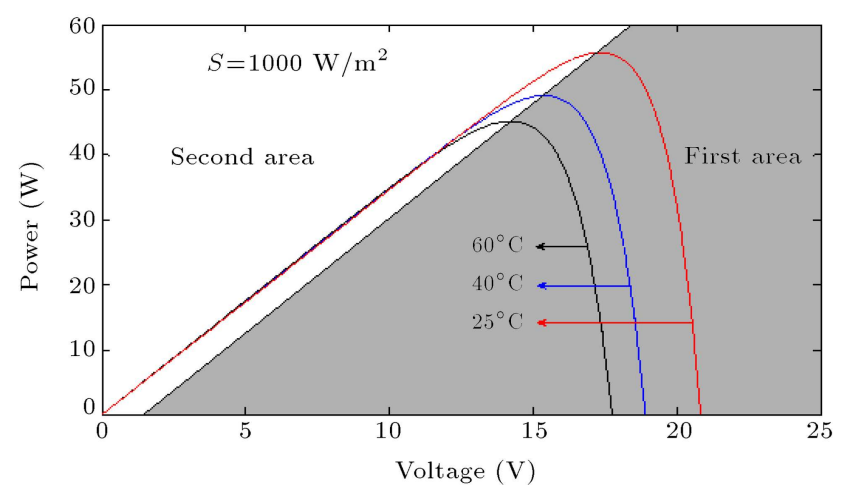

Figure 8. Two functional areas of photovoltaic (PV) curve; $S$ is irradiation.
Table 3. The values of proper gain in $S=900 \mathrm{w} / \mathrm{m}^{2}$.

\begin{tabular}{ccccccc}
\hline \multirow{2}{*}{ Gain } & \multicolumn{6}{c}{ Details of the fitness function } \\
\cline { 2 - 7 } & $\boldsymbol{M}_{\boldsymbol{m}}$ & $\mathbf{S E}$ & $\boldsymbol{t}_{\boldsymbol{p}}$ & $\boldsymbol{V}$ & $\boldsymbol{F i t}$ & $\boldsymbol{T}$ \\
\hline 3.1 & 1.3607 & 0.5835 & 0.0273 & 0.9411 & 0.1018 & $-3^{\circ} \mathrm{C}$ \\
3.2 & 0.7900 & 0.5637 & 0.0246 & 0.2612 & 0.0503 & $7^{\circ} \mathrm{C}$ \\
3.9 & 0.9333 & 0.4929 & 0.0191 & 0.5624 & 0.0492 & $17^{\circ} \mathrm{C}$ \\
3.4 & 0.4796 & 0.4383 & 0.0183 & 0.2906 & 0.0311 & $27^{\circ} \mathrm{C}$ \\
5.7 & 0.3421 & 0.3232 & 0.018 & 0.8308 & 0.1785 & $37^{\circ} \mathrm{C}$ \\
7 & 0 & 0.2299 & 0.1255 & 0 & 0.0577 & $47^{\circ} \mathrm{C}$ \\
1 & 0.0003 & 0.1127 & 0.0653 & 0 & 0.0147 & $57^{\circ} \mathrm{C}$ \\
\hline
\end{tabular}

Table 4. The values of proper gain in $S=600 \mathrm{w} / \mathrm{m}^{2}$.

\begin{tabular}{ccccccc}
\hline \multirow{2}{*}{ Gain } & \multicolumn{6}{c}{ Details of the fitness function } \\
\cline { 2 - 7 } & $\boldsymbol{M}_{\boldsymbol{m}}$ & $\mathbf{S E}$ & $\boldsymbol{t}_{\boldsymbol{p}}$ & $\boldsymbol{V}$ & Fit & $\boldsymbol{T}$ \\
\hline 4.2 & 1.5037 & 0.443 & 0.0181 & 0.04527 & 0.0447 & $-12^{\circ} \mathrm{C}$ \\
4.7 & 1.5230 & 0.4052 & 0.0182 & 0.4864 & 0.0463 & $-2^{\circ} \mathrm{C}$ \\
4.4 & 0.7485 & 0.4056 & 0.0165 & 0.1726 & 0.0253 & $8^{\circ} \mathrm{C}$ \\
4.9 & 1.6606 & 0.3853 & 0.0232 & 1.2856 & 0.0968 & $18^{\circ} \mathrm{C}$ \\
5.3 & 1.3744 & 0.3689 & 0.0224 & 0.9013 & 0.0723 & $28^{\circ} \mathrm{C}$ \\
5.4 & 0.9297 & 0.3574 & 0.0208 & 0.3833 & 0.0405 & $38^{\circ} \mathrm{C}$ \\
5.3 & 0.3943 & 0.3480 & 0.0232 & 0.1111 & 0.0259 & $48^{\circ} \mathrm{C}$ \\
6 & 0.2123 & 0.3084 & 0.0239 & 0.0232 & 0.0184 & $58^{\circ} \mathrm{C}$ \\
\hline
\end{tabular}

system conditions. The flowchart of Figure 7 includes both the regulation of gain and modification of the membership functions in a simulation, while these two regulations are separated in Tables 3 and 4 and the regulation of gain has been performed first. Tables 3 and 4 illustrate the values of Eq. (3) for two irradiation levels of 600 and $900 \mathrm{~W} / \mathrm{m}^{2}$.

Considering the criterion function, if the responses obtained for the values smaller than 0.04 are acceptable, then, according to the data in Tables 3 and 4 , it is distinguished that this condition does not apply to some temperatures. In this case, the change frame of membership functions can be regulated provided that the criterion function is satisfied, and a better answer can be achieved. For example, in Table 3 and at the temperature of $37^{\circ} \mathrm{C}$, the maximum value of the criterion function to have an optimal response is not estimated. Therefore, we will enlarge the value of the criterion function to 0.0277 by modifying the membership functions. Figure 9 shows the output power obtained from photovoltaicat no-control mode, fuzzy control with regulation of gain, and finally with fuzzy control, regulation of gain, and modification of membership functions.

In Figure 9, it can be observed that when the regulation of gain is carried out, the power output fluctuations are undesirably increased despite having a more favorable response rate and a better average 


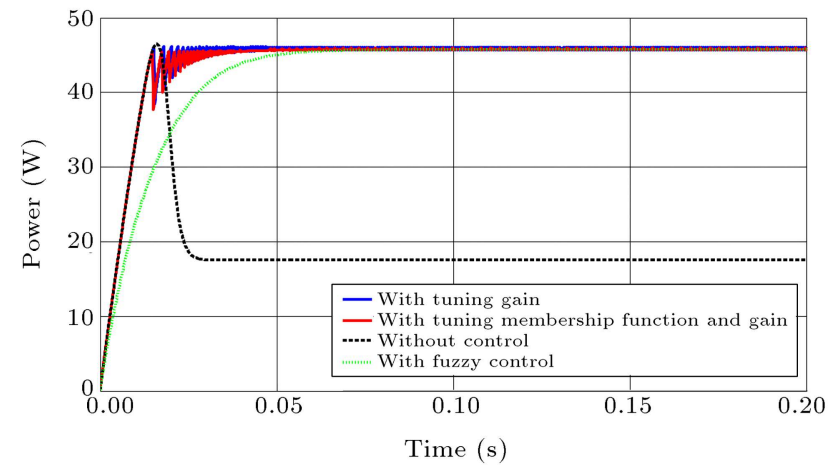

Figure 9. Comparison between the output powers obtained from photovoltaics (PV).

tracked power. In this case, the power fluctuations variance is 0.8308 and the power fluctuations are $11 \%$ of its maximum power. By modifying the membership functions, the volume of fluctuations will decrease acceptably. In this case, the output power variance will be 0.1624 and the power fluctuations get to about $5 \%$ of its maximum power. However, it will lead to the reduction of the system response rate and the power output mean. Then, the steps of regulation of gain and modification of membership functions for different levels of irradiation and ambient temperature in offline mode can be summarized as follows:

1. First, we specify our criterion function according to the standard condition. This condition determines the value of the tracked error of output power, the volume of acceptable fluctuations around the maximum point, as well as the tracking rate;

2. By changing the input gain in the way of the error value and the derivative of input error, the amount of optimal gain will be calculated to satisfy the above criterion for the desired levels of irradiation and temperature. To put it in simple words, for the intended irradiation and temperature, we have obtained a gain that will satisfy the criterion function conditions;

3. At the next stage, for each constant environmental condition, if various gain values do not lead to the satisfaction of the criterion function condition, we select the gain which will be followed by the best answer to the criterion function and modify the membership functions in these conditions in order to authenticate the criterion function conditions. If, despite the modification of membership functions, we could not satisfy the criterion function condition, we consider the best answer which is closer to the conditions of Eq. (3).

\section{Real-time regulation}

By changing the values of irradiation and temperature in a short period of time, maximum power tracking via fuzzy controller in the photovoltaic array will encounter problems and, consequently, the tracking efficiency will be decreased. In this section, using the data in Sections 4 and 5, we want the multiplied gain by the fuzzy controller to be changed proportionate to any variations in the atmospheric conditions, in order to achieve the optimal response. For this purpose, in a few irradiations from 200 to $1000 \mathrm{~W} / \mathrm{m}^{2}$, the appropriate gain will be determined for different temperature changes in each irradiation according to the algorithm in Figure 7. Consequently, a set of gains will be obtained for a particular irradiation and temperature. To calculate the gain value for every atmospheric condition, another fuzzy controller, called decisionmaking fuzzy controller, is required to be used in order to estimate the gain in accordance with the data in Sections 4 and 5 . In this case, the fuzzy controller inputs will be the temperature and irradiation and its output will be the gain proportionate to the two inputs. Here, first, we define the membership functions for this controller. Figure 10(a) describes the membership

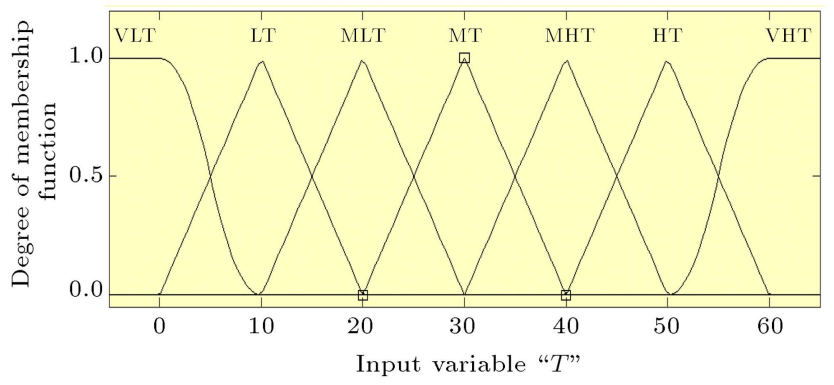

(a)

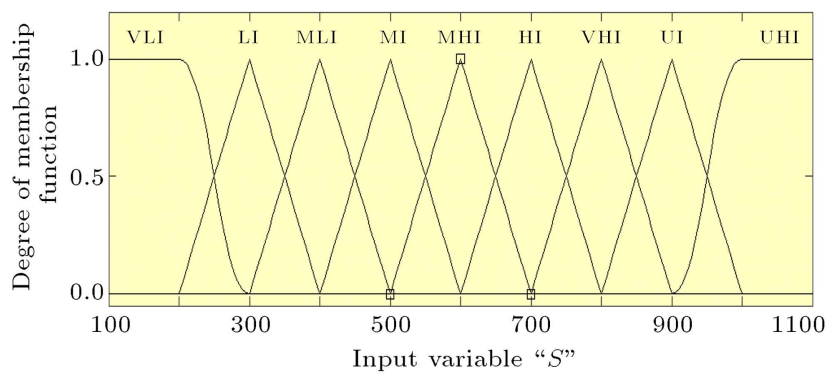

(b)

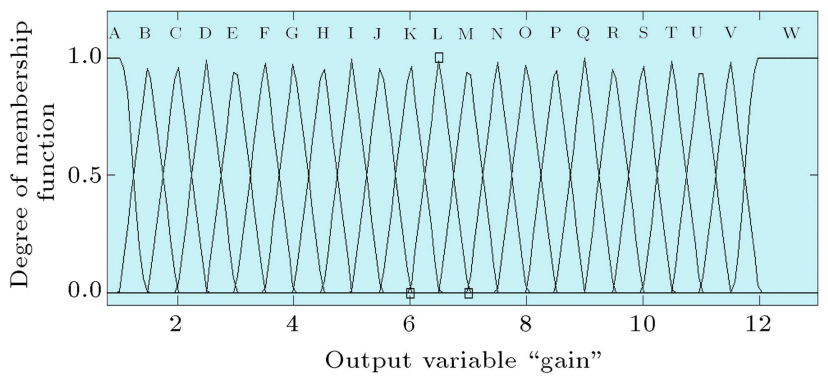

(c)

Figure 10. Membership function of decision-making fuzzy controller: (a) Input temperature; (b) input irradiation; and (c) output gain. 
functions of temperature input, consisting of seven triangle membership functions defined as follows:

VLT: $\quad$ Very Low Temperature;

LT: $\quad$ Low Temperature;

MLT: $\quad$ Medium Low Temperature;

MT: $\quad$ Medium Temperature;

MHT: Medium High Temperature;

HT: High Temperature;

VHT: Very High Temperature.

Irradiation input membership functions consist of nine triangle members defined as follows, which can be observed in Figure 10(b):

VLI: $\quad$ Very Low Irradiation;

LI: $\quad$ Low Irradiation;

MLI: $\quad$ Medium Low Irradiation;

MI: $\quad$ Medium Irradiation;

MHI: Medium High Irradiation;

HI: High Irradiation;

VHI: Very High Irradiation;

UI: Ultra Irradiation;

UHI: Ultra High Irradiation.

Figure 10(c) illustrates the output membership functions, consisting of 23 membership functions named from $A$ to $W$. The decision-making controller rules will be designed using the data in Section 5. Like Tables 3 and 4 , we consider some tables for the irradiation levels of $200,250,300, \ldots, 1000$ and design the controller rules according to the values obtained from these tables. Table 5 shows the set of rules of the decision-making fuzzy controller.

To evaluate our function, we need to perform our simulations for different atmospheric conditions and the variable which is associated with changes in temperature and irradiation, and compare it with the conventional fuzzy controller. It is worth mentioning that the answers obtained from these simulations do not necessarily satisfy the conditions in Eq. (3), because the membership functions will not be performed online. But in this state, regardless of fluctuations around the MPP, increase in the tracking speed and tracking error is reduced. The real-time regulations of the membership functions will undesirably reduce tracking rate of the fuzzy controller. The defuzzifier used in Maxima decision-making controller is of Maximum-Maximum type and it is used due to its simplicity and its low calculation volume compared with the Center Of Gravity (COG) defuzzifier. If the irradiation and temperature changes are the same as those in Figure 11, the tracked power for two modes, one with fuzzy control and the other with adaptive fuzzy control, will be the same as that presented in Figure 12.

According to the performed simulations, it can be concluded that in the conditions where climate changes have undesirable effects on the MPP tracking in fuzzy control, such as reduction of the tracked

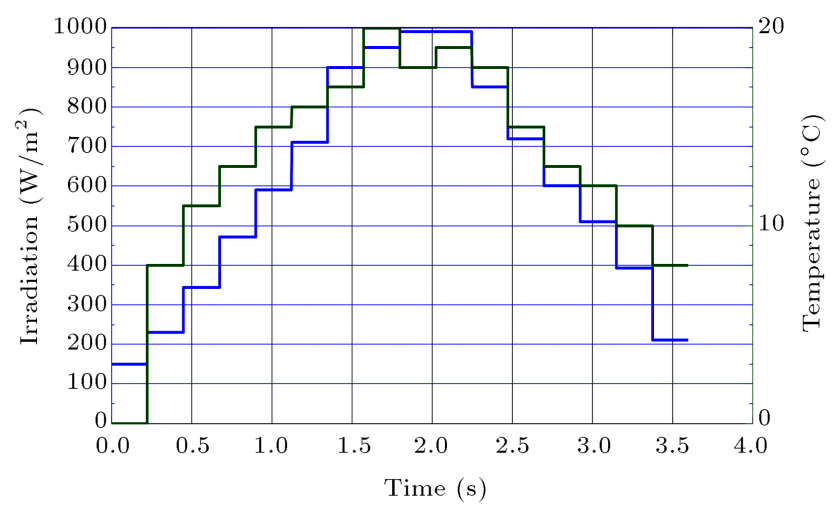

Figure 11. Change of irradiation and temperature with time.

Table 5. Rule base of decision-making controller.

\begin{tabular}{cccccccc}
\hline \multirow{2}{*}{ Irradiation $(\boldsymbol{S})$} & \multicolumn{7}{c}{ Temperature $(\boldsymbol{T})$} \\
\cline { 2 - 7 } & VLT & LT & MLT & MT & MHT & HT & VHT \\
\hline VLI & W & W & W & W & W & W & W \\
LI & P & O & R & P & T & Q & R \\
MLI & M & L & M & N & N & N & O \\
MI & J & J & J & L & M & L & N \\
MHI & H & I & J & J & I & J & K \\
HI & G & K & H & H & I & K & N \\
VHI & F & F & G & H & K & O & A \\
UI & E & E & G & H & J & S & A \\
UHI & E & F & I & P & A & A & A
\end{tabular}

Note: $T$ : temperature; $S$ : irradiation; $M$ : abbreviation of medium; $V$ : very;

$L$ : low; $H$ : high; $U$ : ultra; and $I$ : irradiation. 


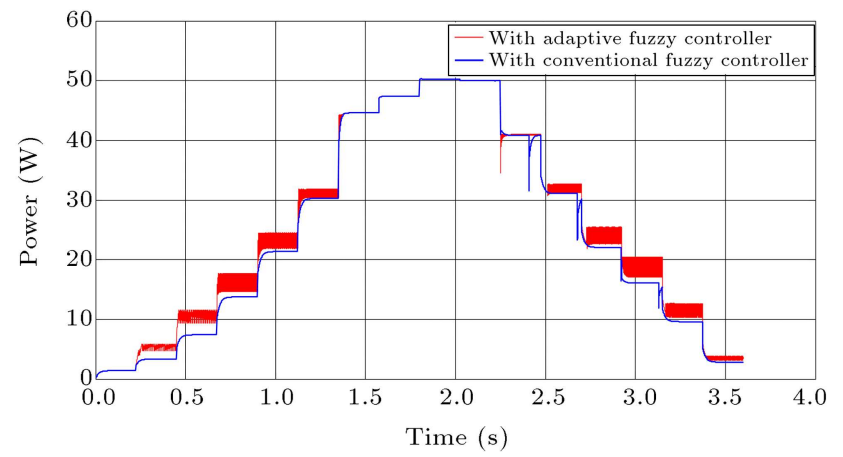

Figure 12. Maximum Power Point Tracking (MPPT) comparison between adaptive fuzzy controller and conventional fuzzy controller.

power, the adaptive fuzzy control has managed to resolve the defects by regulating the gain efficiency, as a result of which the maximum output power will be tracked more efficiently. However, this method has some deficiencies, including using two more sensors for measuring temperature and irradiation as well as complexity of controller.

\section{Conclusion}

When the temperature and radiation intensity can be changed independently, MPPT can be difficult with fuzzy controllers. As it is already mentioned in Section 4 , the fuzzy controller is designed for standard conditions and actually has an acceptable function in the conditions close to standard. But when there are many changes compared with the standard conditions (for example, if the irradiation reaches from $300 \mathrm{~W} / \mathrm{m}^{2}$ to $1000 \mathrm{~W} / \mathrm{m}^{2}$, instantaneously), there will be lots of errors in tracking. For decreasing this error is added another fuzzy controller (decision-making controller) which tunes gain of inputs of fuzzy controller. The simulation results, in this case, indicate that the maximum output power of solar cells is tracked with less error. However, the proposed method also has some deficiencies, including using two more sensors for measuring temperature and irradiation as well as using another fuzzy controller for a better tracking, which lead to a more complex system.

\section{References}

1. Buresch, M., Photovoltaic Energy Systems Design and Installation, 2nd Ed., McGraw-Hill, New York, USA (1983).

2. Femia, N. and Petrone, G. "Optimization of perturb and observe maximum power point tracking method", IEEE Power Electron, 20, pp. 963-973 (2005).

3. Wolfs, P. and Quan, Li. "A current-sensor-free incremental conductance single cell MPPT for high performance vehicle solar arrays", Power Electronics
Specialists Conf. (PESC), 37th Annual IEEE, pp. 1-7 (2006).

4. Wolf, S. and Enslin, J. "Economical PV maximum power point tracking regulator with simplistic controller", Power Electronics Specialists Conf. (PESC), 24th Annual IEEE, pp. 581-587 (1993).

5. Umeda, F., Ohsato, M.H., Kimora, G. and Shioya M. "New control method of resonant dc-dc converter in small scaled photovoltaic system", Power Electronics Specialists Conf. (PESC), 23th Annual IEEE, pp. 714718 (1992).

6. Dorofte, C., Borup, U. and Blaabjerg, F. "A combined two-method MPPT control scheme for grid-connected photovoltaic systems", Power Electronics and Applications, European Conference, pp. 1-10 (2005).

7. Chen, Y.Y. and Man, Y.K. "Constant current-based maximum power point tracking for photovoltaic power systems", EDT from IEEE, pp. 3422-3425 (2010).

8. Mutoh, N. and Matuo, T. "Prediction-data-based maximum power point tracking for photovoltaic power generation system", IEEE Power Electron Conference, In Proc. 33rd Annu, pp. 1489-1494 (2002).

9. Li, J. and Wang, H. "Maximum power point tracking of photovoltaic generation based on the optimal gradient method", IEEE, pp.1-4 (2009).

10. Altas, I.H. and Sharaf, A.M. "A novel maximum power fuzzy logic controller for photovoltaic solar energy systems", Renewable Energy Journal, 33, pp. 388-399 (2008).

11. Xiangdong, Q. and $\mathrm{Li}, \mathrm{H}$. "Application of fuzzy logic and immune response feedback for PV generating system", IEEE, pp. 119-124 (2007).

12. Bouchafaa, F., Hamzaoui, I. and Hadjammar, A. "Fuzzy logic control for the tracking of maximum power point of a PV system", Energy Procedia, 6, pp. 633-642 (2011).

13. Patcharaprakiti, N., Premrudeepreechacharn, S. and Yosanai, S. "Maximum power point tracking using adaptive fuzzy logic control for grid-connected photovoltaic system", Renewable Energy, 30, pp. 1771-1788 (2005).

14. Feng, D. and Junlei, M.A. "A MPPT PV system control method based on fuzzy neural network", Computing Technology and Automation, 26, pp. 25-28 (2007).

15. Moradi, H.M., Tousi, S.M.R., Nemati, M., Basir, N.S. and Shalavi, N. "A robust hybrid method for maximum power point tracking in photovoltaic systems", Solar Energy, 94, pp. 266-276 (2013).

16. Li, F., Shi, P., Wu, L. and Zhang, X. "Fuzzy-modelbased D-stability and non-fragile control for discretetime descriptor systems with multiple delays", IEEE Trans. on Fuzzy Systems, 22(4), pp. 1019-1025 (2014).

17. Wang, Y. and Fei, J. "Adaptive fuzzy sliding mode control for PMSM position regulation system", International Journal of Innovative Computing, Information and Control, 11(3), pp. 881-891 (2015). 
18. Green, M.A. "General solar cell curve factors including the effects of ideality factor. Resistance solid-state electron", Temp., Ser., 20, p. 265 (1977).

19. Enrique, J.M., Duran, E., Sidrach-de-Cardona, M. and Andujar, J.M. "Theoretical assessment of the maximum power point tracking efficiency of photovoltaic facilities with different converter topologies", Solar Energy, pp. 31-38 (2007).

20. Elmas, C., Deperlioglu, O. and Sayan, H.H. "Adaptive fuzzy logic controller for dc-dc converters", Elsevier, Expert Systems with Applications, pp. 1540-1548 (2009).

21. Gheibi, A., Mohammadi, S.M.A. and Maghfoori, M.M. "Maximum power point tracking for solar cell systems by using adaptive fuzzy logic controller", 26th International Power System Conf. (PSC), pp. 1-7 (2011).

22. Gheibi, A., Mohammadi, S.M.A. and Maghfoori, M.M. "Comparing performance of PID and fuzzy controllers in the present of noise for a photovoltaic system", Journal of Mathematics and Computer Science (JMCS), pp. 69-76 (2014).

23. Zadeh, L.A. "Fuzzy logic = computing with words", IEEE Trans, Fuzzy System, 4, pp. 103-111 (1996).

24. Wang, L.X., A Course in Fuzzy Systems and Control, 2nd Ed., New Jersey, Prentice Hall, USA (1997).

25. Astorm, K.J. and Wittenmark, B., Adaptive Control, 2nd Ed., Boston, Addison-Wesley Longman Publishing Co. Inc., USA, pp. 1-2 (1994).

\section{Biographies}

Amir Gheibi received his BS degree in Electrical Engineering in 2007, Tehran, Iran, and MSc degree from Shahid Bahonar University of Kerman, Iran, in 2012. He is currently PhD student at Tabriz University. His areas of interest and experience are reinforcement learning, adaptive intelligent systems, fuzzy systems, and robust control.

Seyed Mohammad Ali Mohammadi received his BS degree in Electrical Engineering from Sistan and Baluchestan University, Zahedan, Iran, in 1997, MSc degree from Esfahan University, Iran, in 2002, and PhD degree in Applied Mathematics in Control Systems from S.B.U.K, Kerman, Iran, in 2009. His research interests are in fuzzy systems, artificial intelligence, instrumentation, and control systems.

Malihe Maghfoori Farsangi was born in Kerman, Iran, in 1972. She received her BS degree in Electrical Engineering from Ferdowsi University, Iran, in 1995 and $\mathrm{PhD}$ degree in Electrical Engineering from Brunel Institute of Power Systems, Brunel University, UK, in 2003. She is interested in robust control, model predictive control, power system control, and computational intelligence. Dr. Farsangi joined the Department of Electrical Engineering, Shahid Bahonar University of Kerman, as an Assistant Professor, in 2003, and she has been a Professor since 2014 . 